\title{
A new renaissance for creativity in technology and the arts in the context of virtual worlds
}

\author{
R. A. Earnshaw ${ }^{1}$ (D) \\ Accepted: 28 May 2021 / Published online: 10 June 2021 \\ (c) The Author(s) 2021
}

\begin{abstract}
Where do new ideas come from and how are they generated? Which of these ideas will be potentially useful immediately, and which will be more 'blue sky'? For the latter, their significance may not be known for a number of years, perhaps even generations. The progress of computing and digital media is a relevant and useful case study in this respect. Which visions of the future in the early days of computing have stood the test of time, and which have vanished without trace? Can this be used as guide for current and future areas of research and development? If one Internet year is equivalent to seven calendar years, are virtual worlds being utilized as an effective accelerator for these new ideas and their implementation and evaluation? The nature of digital media and its constituent parts such as electronic devices, sensors, images, audio, games, web pages, social media, e-books, and Internet of Things, provides a diverse environment which can be viewed as a testbed for current and future ideas. Individual disciplines utilise virtual worlds in different ways. As collaboration is often involved in such research environments, does the technology make these collaborations effective? Have the limits of disciplinary approaches been reached? The importance of interdisciplinary collaborations for the future is proposed and evaluated. The current enablers for progressing interdisciplinary collaborations are presented. The possibility for a new Renaissance between technology and the arts is discussed.
\end{abstract}

Keywords Creative processes $\cdot$ Virtual design $\cdot$ Virtual exhibition $\cdot$ Cyberworlds $\cdot$ Social media interactions $\cdot$ Paradigm shift

\section{Communication}

Creation and communication have been two fundamental activities of humans from the beginning of civilisation. Cave paintings are artefacts which include both of these aspects. They also reflect the context and culture of civilisations at the time to some degree, as well as the availability of appropriate raw materials with which to create. Creation and communication have therefore two-way aspects, in expressing ideas in the minds of the creators to others, and also reflecting back to the creators an image of what has been created. In addition, a created work also communicates to future observers who were not part of the original context. It tells a story today about what happened in the past. This story may be subject to a variety of interpretations, but it is

R. A. Earnshaw

r.a.earnshaw@bradford.ac.uk

1 Department of Computer Science, Faculty of Engineering and Informatics, University of Bradford, Bradford, UK still a story. It is a communication associated with the created work.

As raw materials and culture change over time, so do the artefacts and images which are produced. The ideas of the creators-the humans involved-also develop and change. This synchronicity and interrelationship tells a continuing story about the artworks and also about their creators. This story may change the initial context and culture into something completely new. The continuing story may also change the culture of subsequent generations over time as they reflect upon what was initially created. Like a stone thrown into a pond, the resulting ripples can spread far and wide.

\section{Changes produced by tools and interaction}

A further aspect is the ways in which the created works which are produced may produce a change in the attitude and outlook of the observer or the creator. Nations may go to 
war against each other because of an image that is regarded as offensive to a particular point of view, or they may sign a peace treaty because they have been motivated to do so by a piece of text or an image.

The impact of created works can therefore be substantial. Newton's third law of motion expressed the principle that for every action there is an equal and opposite reaction. Although this was initially framed in the context of the physical world, it is equally applicable to the creative and cultural worlds.

The expression of human creativity has depended to a large extent on the availability of appropriate tools. Over time these tools have developed from producing images into text, writing, and reading. And also producing 3D images and objects such as sculptures. For example, Michelangelo created drawings, paintings, and sculptures which communicated his interpretations of various ideas and scenes. The tool that is available at the time may affect the created work that is produced to some degree. If it was to be repeated today with a different tool, it may produce a completely different result. But today is also a different era and the cultural context could affect the way the tool is used. Thus, a tool is not just an object in the hand of the creator, it is also an integral part of the environment in which the created work is produced.

Can an observer of a created work interact with it and change it? Traditionally, the static works of artists and sculptors have been regarded as sacrosanct- they are their works and should not be changed by others. This has been carried on as a tradition to text, books, and drawings. An inherent copyright on the original work has been regarded as belonging to its creator.

However, an artist today may create a physical artwork or exhibition where the interactions and interventions by the public are invited. These can change the original work into something new. It therefore becomes a dynamic created work which reflects not just the creativity of the artist but of all those who subsequently interact with it. Over time it becomes a different artwork to the one the artist originally produced.

\section{The origin of new ideas}

But where does a new idea for a created work come from? Does it come from the mind of creator? Does it come from an observation of something in the natural world? (as appears to be the case with cave paintings). Is it stimulated by the availability of a new tool? Is it an expression of some social or cultural aspect of the environment of the creator? It could be a complex blend of some, or all, of these factors. Figure 1 shows a lightbulb as a representation of a new idea moment. A key idea here is illumination, that is to

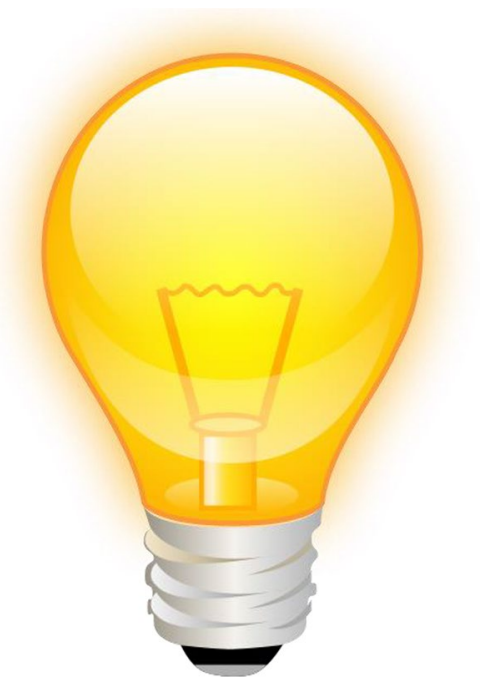

Fig. 1 A picture of a lightbulb is associated with someone having an idea, a sign of creativity. This file is licenced under the Creative Commons Attribution-Share Alike 3.0 Unported licence. https://en. wikipedia.org/wiki/Creativity

say, something is now visible and clear that was previously obscure or hidden.

\section{Does technology produce a paradigm shift?}

Figure 1 has used technology to produce this image. Thus, in today's world, we must add technology to the array of possible tools which may be used to produce created works. In particular, digital tools and environments are being used in various ways in the creative process. The artist David Hockney used an iPad to create a series of images of landscapes which have been displayed in various national and international exhibitions. Technology can therefore be an artistic tool, but is it a tool which enhances the human creative process, or is it shaping and constraining them in ways that we are not aware of? Can technology be limiting our ability to be fully creative?

In order to answer this question, we need to consider the ways in which digital technology may be utilised in the creative process.

\subsection{Technology as computation}

The power of the digital computer can be used to process algorithms to produce a variety of lines, colours, and shapes which then be combined into pictures, and output to a printing device. Such digital art has been displayed in exhibitions either alongside traditional art, or in a section on its own. In terms of traditional exhibitions devoted to the works of 
the humans who created them, it is not fully clear who is the creator of a digital work. However, although the computer processed the data and generated the digital art, it was a human who designed the original algorithm on which the computation is based. Therefore, the computer can be regarded as a tool in the hands of a human creator, just as the iPad was a tool in the hands of David Hockney. His works were exhibited in traditional art exhibitions. It may be the case that the more famous the artist is, the more acceptable are the tools that they choose to use. All new tools need pioneers to show how they may be used, before they can become fully normalised and accepted.

\subsection{Technology as synthesiser}

Digital computers can combine many different streams of information into one-whether from an external input device, or generated internally by a computer programme. They can therefore generate new artificial artworks and worlds. The human controller of this process may utilise a set of parameters to vary the output produced by this process, thus giving different artworks or different virtual worlds. The latter are new kinds of worlds which may reflect the characteristics of the natural, real world, or they may be entirely new. They express the imagination of the human creator.

\subsection{Technology as interactor}

Such synthetic worlds are artificial to a large degree, but they can be utilised by artists and designers, and also allow humans to interact with them. If the virtual world represents an architect's drawing of a building, a designer may wish to change portions of the drawing in order to enhance the external appearance of the building, or to increase the usability of the space within the building. In this case, the user is controlling the interaction and the effect that it has on the overall design process.

\subsection{Technology as integrator}

Multiple forms of input and also technology can be brought together on digital platforms to integrate text, speech, image, and video.

\subsection{Technology as automaton}

A set of coded instructions may be supplied to a computer which then follows them. An example of this is a robot. An automated car manufacturing plant may use many robots to implement the predetermined processes required to produce a car. Such processes are fixed but they may be optimised by the human who designed the original instructions, if the factory wishes to see if it can produce more cars in a certain time, or more cars of a particular type.

Therefore, technology may not be limiting the human's creative processes, provided it is used in ways which demonstrate an understanding of its advantages and limitations. Misuse of any tool can hamper the creative process, and produce outputs which are of little or no value.

\subsection{A paradigm shift?}

Does the use of new technology constitute a paradigm shift for creativity in the context of these digital environments?

A synthetic world, which is a digital world and therefore artificial, may represent an opportunity to explore something new for those who interact with it. It could stimulate new ideas and new creative processes which would not have been possible in the real world. Therefore, the digital environment can generate something that is new. Its potential appears to be only limited by the imagination of the person generating it, or interacting with it.

A new approach which utilises new tools may gain a new set of people who are devoted to its development and exploitation. This is therefore something that would not have happened without the new approach.

In addition, the volume of information which such a digital environment requires is immense, and many orders of magnitude greater than that of the natural world. The invention of the printing press in the fifteenth century demonstrated a similar capacity to communicate large amounts of information to a large number of people in ways that were not possible using the traditional methods. Digital environments can have a similar transformative effect in today's world. It may therefore be regarded as a new technological paradigm.

Kuhn's concept paradigm shift [1] was largely proposed by observing the effects caused by the Scientific and Industrial Revolutions in the sixteenth and seventeenth centuries. In this period, which was often denoted as the Age of Enlightenment, developments in mathematics, physics, astronomy, biology, chemistry, and engineering enabled new products and services to be supplied to human populations which saved unnecessary labour and produced greater efficiencies in manufacturing, communication, and transport processes. It also fundamentally changed the understanding of society about nature, and about how it was composed, and how it could be utilised.

Computers provide the basic computation to generate virtual worlds, and the Internet provides the connectivity to bring people and digital worlds together. Computers have produced greater efficiencies in manufacturing, communication, and transport processes. They may not have fundamentally changed our understanding of the natural world at the macro level (as the Scientific Revolution 
did), but they are providing a finer and more detailed level of information and understanding in areas such as DNA, genetic engineering, quantum theory, the structure of matter, the origin of the universe. This has been made possible by the real-time processing and visualisation of large amounts of data (the so-called Big Data challenges) and the involvement of interdisciplinary teams of researchers in national and international Data Centres.

Thus, although there are clear differences in the transition from the medieval era to the scientific era, the extent of today's changes may well constitute a new Renaissance when viewed with hindsight. This may become more apparent over time as the extent of the current transformations is more fully understood.

What are the key aspects of the digital revolution that most affect creative processes in particular, and society in general? It is proposed that connectivity and communication are key aspects to be explored further.

\section{Exploring creativity}

Creativity may be defined as the use of imagination to innovate and produce something new, or the generation of some original ideas which result in a new approach, or new inventions. It may depend upon expertise if the domain being considered is one where some form of technical skill is required (e.g. digital environments). It may also depend on motivation and opportunity. For example, an artwork may require many months of dedication and iteration on the part of the artist in order to produce the final work. In a new area, such as virtual worlds, it may depend on the opportunity to work in the new area and the availability of appropriate facilities.

The value of creativity can depend on a number of factors and the particular application area. Artworks and sculptures can depend upon their aesthetic value. A book may depend upon the genre to which its content belongs. Producing new forms of virtual worlds may depend upon their form and function, and also their ease of use.

How may the processes of creativity be communicated? It could be directly-by the created work itself. A painting or sculpture is usually intended to be a freestanding and unique expression of the artist's concept. It may be subject to a variety of interpretations both at the time of its creation, and also subsequently. In addition, the creator may describe in words or a sequence of images what they are seeking to do as their artwork is being created. Also, the final work may have an aesthetic value which goes beyond the initial ideas and circumstances which created it.

\section{Virtual worlds and creativity}

Virtual worlds can bring creators and collaborators together into one environment when they are working in partnership on a particular task or object. The public may also be included as viewers or interactors. The creators may wish to include modifications on the created work which are supplied by the public.

A virtual world can be a walk-in environment as shown in Fig. 2, where sensors which are built into the physical world of the Cave provide real-time information to the computer(s) which are generating the content of the virtual world as experienced by the user. For example, as the user moves in the virtual world, the visual world changes accordingly. The user can also have a hand-held device (linked to the computer) which they are able to use to provide different kinds of direct interaction to steer, or edit, aspects of the world they can see. If this virtual world contains an artwork, then the user-as-artist can modify the artwork in shape or colour with immediate effect. However, it is clearly important for the interactive device to be tuned to the artist's particular way of working. That is, it should facilitate the artist's creative processes and not inhibit them.

It is possible that the name of this virtual world (Cave) is an allusion or reference to the Cave in Plato's Republic. This is an artificial world in which a philosopher is invited to use their imagination to distinguish between perception, reality, and illusion. For example, people who are chained to face a blank wall of the Cave can see the

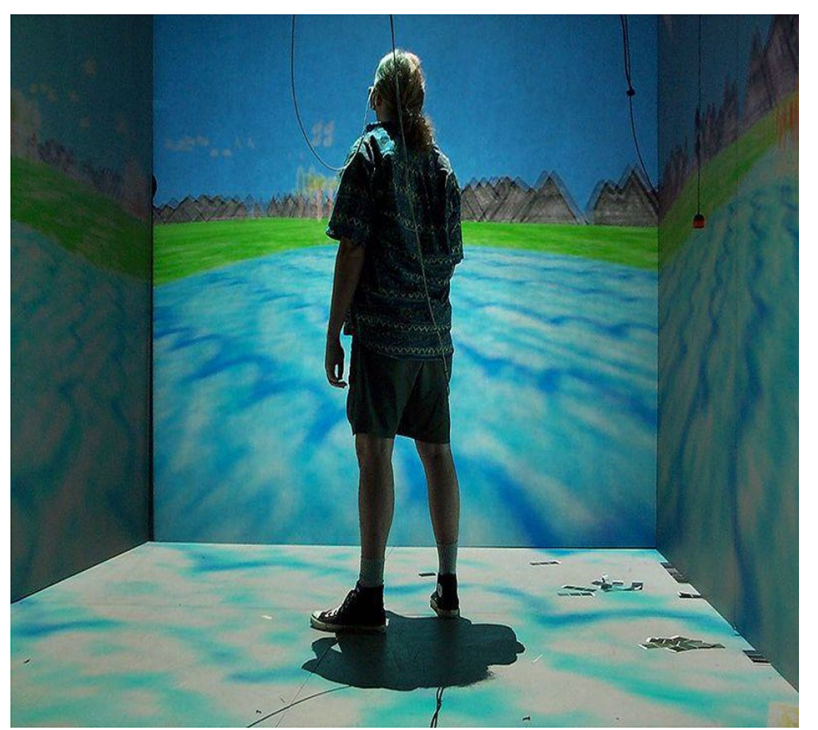

Fig. 2 A cave digital world. "CAVE Crayoland" by User:Davepapeown work (self-photograph using timer). Licenced under public domain via commons. https://commons.wikimedia.org/wiki/File: CAVE_Crayoland.jpg\#/media/File:CAVE_Crayoland.jpg 
shadows projected on to the wall from objects passing in front of a fire behind them. If the prisoners know of no other world than the Cave, these shadows become to them the reality of the world behind them and external to the Cave. However, they are not accurate representations of the real world. Therefore, a philosopher should seek to perceive and understand higher levels of reality, otherwise they will continue to see the real world only in the terms of those who are prisoners within it, and by doing this they become prisoners themselves.

Although this is a virtual world of human imagination, it is not an allegory or representation of a digital virtual world. Although this digital world is also artificial, it is, nevertheless, a real construct within which a model of the real world is able to be built and within which the user can move and interact in an analogous way to the real world. Therefore, it is not a philosophical construct as in Plato's Cave.

In addition, research in architectural design in immersive virtual environments has shown that designers perceive and understand volumes, spaces, and spatial relationships better than in 2D environments such as those expressed in architectural drawings [2]. This is because users in virtual worlds are in what is perceived as a $3 \mathrm{D}$ world even though it is composed of 2D images with added depth cues- to simulate the three dimensions as they are seen in the real world. This can also assist designers and users to explore this 3D world, or to gain an understanding of which parts of the 3D world are likely to be more useful to examine in more detail than others. This can make the design process more efficient and effective overall, thus saving time and money.

A virtual world also enables simulations to be done. A flight simulator is an example of a virtual world dedicated to one application domain, which is the training of aircraft pilots. The virtual world replicates the images and forces of the real world to a high degree of accuracy in order to act as an equivalent to the flying of the aircraft in the real world. This equivalence is subject to a certification process.

The training of the pilot in the simulator can check that they are able to perform all the normal manoeuvers expected in the flying of the aircraft. It can also subject pilots to react with extraordinary manoeuvers, of the kind required in an emergency situation. These would be more difficult to do, or perform safely, with a real aircraft. The simulated world is therefore able to do more than would be practically possible in the real world.

Similarly, the utilising of virtual worlds for creative processes is able to generate new kinds of content which are not possible in the real world. In this sense, they are more like the virtual Cave envisaged by Plato. Such worlds can be generated by computer programmes using artificial intelligence techniques and enable creative works to be edited at a much faster pace than would be possible in the real world.
Two case studies are now described which show how collaborative virtual worlds can be used to achieve particular objectives.

A Virtual Entertainment Park (VPARK) [3, 4] was a European R\&D project which used virtual humans in a collaborative virtual world to represent real-world actors in a number of application scenarios. This enabled the actors who were separated geographically to come together in the virtual world in real-time. Two application scenarios were investigated: theatre rehearsal and orchestra rehearsal. Virtual theatre rehearsal was found to be particularly useful for those aspects of the rehearsal processes which had to be planned in advance, such as the positions of the actors on the stage, and their movements relative to each other as a particular scene was being played out. It also gave the actors additional opportunities to memorise the script and to see it enacted in the context of the virtual stage. Savings in travel time and costs were achieved compared to the traditional rehearsal processes.

A further project VISTA (Virtual Interactive Studio Television Applications using Networked Graphical Supercomputers) was designed to create interactive broadcast drama by bringing together content creators and consumers together [5, 6]. This project offered the capabilities of mixing real and synthetic objects and characters in any combination, depending on the circumstances, and controlled remotely by TV viewers. Broadcasting companies could develop and execute interactive TV programmes where the images are constituted in virtual environments and in which the viewers could actively participate and direct the programme being transmitted.

Three application scenarios were tested using the system:

- An interactive television drama (simultaneously on three channels) with viewer participation

- Creation of a virtual presenter who could be controlled remotely in real-time.

- Creation of an interactive virtual driving environment to teach traffic rules.

This environment could be considered as analogous to a physical studio where the inputs and outputs are controlled by content creators and the viewers.

\section{Human creativity}

Using digital technology to reframe the creation and access of artistic works has resulted in the consideration of the human processes involved in creativity. This is analogous to the ways in which virtual learning environments have resulted in more detailed considerations of how humans learn in real-world environments. It may also be compared 
with the use of artificial intelligence techniques and whether they mirror those of human intelligence. Are they the same as the normal human processes (but perhaps at increased speed and with more facility for interaction) or are they qualitatively and quantitatively different? That is, do they replicate the normal human processes, or are they capable of offering something new? This is still under discussion [7-10].

The digital environment has enabled content creators and users to see a greater variety of options before homing in on their preferred content. This could stimulate creativity for some, but it may not be true for all. It could depend on the circumstances and the types of content being created. However, they do provide the artist with a greater variety of tools and facilities than are normally available with manual methods.

In addition, more power and capability may be available in the future. It is difficult to predict how virtual worlds will develop, but it is common in new fields to underestimate their potential [11].

\section{Enhancing creativity}

Projects which bring together artists, social media and scientists are summarised by Liggett et al. [12] and Earnshaw et al. [13]. They all involve collaboration to some degree, and they all have delivered added value to the projects. Thus collaboration, particularly interdisciplinary collaboration, does appear to deliver significant benefits.

Design rationale and creativity can be mutually supportive [14]. New designs often open up new opportunities and new ways of thinking.

It is also possible that digital environments will enable new types of content to be produced that are not currently envisaged, and therefore surpass the current capabilities of humans $[15,16]$.

\section{Interaction with audiences}

An online art exhibition (Fig. 3) may be designed with the intention of interacting with its audiences, and in turn may incorporate these interactions into its future content. This produces an exhibition which is dynamic and transcends the initial content and space. Because it is online, it is available globally and crosses national and cultural boundaries [17, 18]. An Art Expo at Wrexham Glyndwr University is an example of both a physical and virtual exhibition $[19,20]$.

Online resources will serve the interests of many users [21]. This massification of online exhibition spaces and users can result in interactions which accelerate innovation, just as crowd-funding facilitates the collection of funds for a

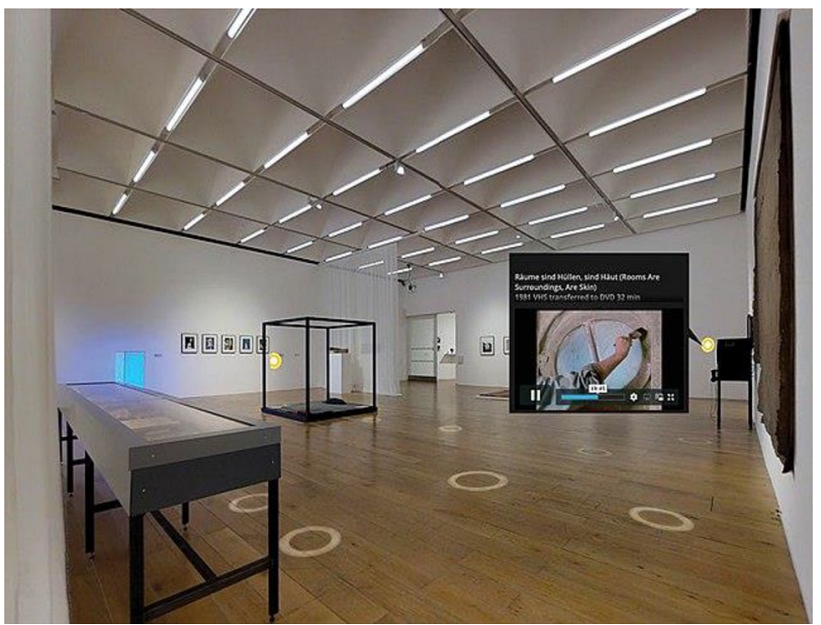

Fig. 3 Virtual exhibition produced by V21 Artspace, The house of fame, convened by linder at nottingham contemporary. This file is licenced under the creative commons attribution-share alike 3.0 unported licence. https://en.wikipedia.org/wiki/Online_exhibition

particular objective in which people have a common interest. In addition, the Internet is an integral part of daily existence. Clark [22] proposed that one Internet year was equivalent to seven calendar years, so a connection to the Internet and the use of its content could substantially advance the processes of development [23]. Social media can also assist in this process $[24,25]$. Such a potentially large volume of people can accomplish substantial change over a relatively short period of time [26-30].

\section{Intrinsic and extrinsic creativity}

Everyone has some form of innate creativity which is capable of development via learning and exploration. However, it does need the opportunity to be able to engage in these activities, and it may not be possible for everyone. When presented with new information we have the opportunity to react to it and develop it further [31].

\section{Can creativity be quantitatively assessed?}

Various parameters can be considered for measuring the results of creativity. These include aesthetics, value, originality, and receptivity by public audiences. In addition, its commercial value over time may be relevant. Guildford [32] used psychometrics. Researchers in this field have produced numerous criteria to seek to measure creativity $[33,34]$. These include uniqueness, complexity, risk taking, and the multiplicity of creative outputs. 


\section{Organisational aspects of creativity}

Some organisations are known to be more open to change than others. Some organisations operate in teams rather than in fixed hierarchies. When a new problem needs to be solved, an appropriate interdisciplinary team is brought together to address it and find ways to reach a solution. When the objective is reached the team is disbanded and the members may re-form into other teams to address different problems. In this way, a reservoir of multi-disciplinary expertise is being built up which is dynamic and adaptable to new situations. It can be argued that this is a more creative approach than an inflexible structure based on history and traditions. New kinds of problem require new kinds of environments and expertise to address them.

Organisations which wish to benefit from creativity and new ideas will seek to implement structures which facilitate this, of the kind previously described. However, this may not produce creative solutions in all cases. It is difficult to predict the outcomes with certainty [35].

Amabile [36] summarised six organisational practices that could effect creativity. These are: support, encouragement, challenge, freedom, resources, and group-working.

The organisational environment may be a combination of physical and digital aspects, and is therefore a hybrid environment to some degree. This makes the situation more complicated [37]. There may also be cultural and ethical issues which increase the complexity $[38,39]$.

\section{Conclusions}

The interrelatedness of creation and communication has been established by observing how artworks have been produced since the dawn of civilisation. Not only do they tell a story by, and through, their content, but they also reflect the culture and context of the environment in which the artist or creator was working. The context can shape the content produced and this content can reflect back a message to the culture in which it was created.

The use of tools has varied over time, culminating in the use of sophisticated interactive facilities in the context of today's digital cyberworlds. Technology has been considered as computation, synthesiser, interactor, integrator, and automaton. The case has been presented for considering the aggregation of these factors as not just content enablers, but more fundamentally as a paradigm shift compared to what was possible in the pre-digital era.

The expression of creativity in cyberworlds has been considered. It has been observed that cyberworlds can bring together content creators and the general public into one entity capable of modifying and reframing the content that is created. Cyberworlds have the potential to enable content creators to interact with global audiences in terms of display, as well as direct interaction, and modification, of the content.

The degree to which creativity may be assessed and measured has been reviewed. In the context of global audiences in cyberworlds, the range of interactors that is possible may be considered as a crowd generating its own distinctive contribution to the ongoing task of content creation.

Future work can include the use of artificial intelligence techniques to handle the more routine aspects of information requests, and also to generate new forms of content not initially envisaged by the creator. In addition, augmented reality can bring new dimensions of experience to users in cyberworlds.

Acknowledgements This paper was presented as an Invited Lecture at Cyberworlds 2020, 29 Sept-1 Oct, Caen, France, but was not published. Some of the concepts in this paper have been published in Earnshaw R.A. (2016): "Research and Development in Art, Design, and Creativity", in Chapter 4 "Creativity and Creative Processes in Art and Design”, Springer, Switzerland ISBN: 978-3-319-33004-4. These are included by permission of the author.

Funding No grants were associated with this work.

\section{Declarations}

Conflict of interest The author declares he has no conflict of interest.

Open Access This article is licensed under a Creative Commons Attribution 4.0 International License, which permits use, sharing, adaptation, distribution and reproduction in any medium or format, as long as you give appropriate credit to the original author(s) and the source, provide a link to the Creative Commons licence, and indicate if changes were made. The images or other third party material in this article are included in the article's Creative Commons licence, unless indicated otherwise in a credit line to the material. If material is not included in the article's Creative Commons licence and your intended use is not permitted by statutory regulation or exceeds the permitted use, you will need to obtain permission directly from the copyright holder. To view a copy of this licence, visit http://creativecommons.org/licenses/by/4.0/.

\section{References}

1. Kuhn, T.: The Structure of Scientific Revolutions, 50th Anniversary edn. University of Chicago Press, Chicago (2012) (originally published 1962)

2. Schnabel, M.A.: Architectural design in virtual environments: exploring cognition and communication in immersive virtual environments. PhD Thesis, University of Hong Kong (2004). http:// hub.hku.hk/handle/10722/28017. Accessed 11 April 2021

3. Virtual Amusement Park (VPARK). http://cordis.europa.eu/proje ct/rcn/46555_en.html. Accessed 11 April 2021

4. Joslin, C., Molet, T., Magnenat-Thalmann, N., Esmerado, J., Thalmann, D., Palmer, I.J., Chilton, N., Earnshaw, R.A.: Sharing 
attractions on the net with VPARK. IEEE Comput. Graph. Appl. 21(1), 61-71 (2001)

5. Virtual interactive studio television application using networked graphical supercomputers (VISTA). http://cordis.europa.eu/proje ct/rcn/32297_en.html. Accessed 11 April 2021

6. Flerackers, C., Earnshaw, R.A., Vanischem, G., Van Reeth, F., Alsema, F.: Creating broadcast interactive drama in a networked virtual environment. IEEE Comput. Graph. Appl. 21(1), 56-60 (2001)

7. Boden, M.: Can computer models help us to understand human creativity?. http://nationalhumanitiescenter.org/on-the-human/ 2010/05/can-computer-models-help-us-to-understand-humancreativity/. Accessed 11 April 2021

8. Boden, M.A.: The Creative Mind: Myths and Mechanisms. Weidenfeld and Nicholson, London (1990)

9. Boden, M.A. (ed.): Dimensions of Creativity. MIT Press, Cambridge (1996)

10. Pearce, M.: Boden and Beyond: the creative mind and its reception in the academic community. http://webprojects.eecs.qmul.ac.uk/ marcusp/notes/boden.pdf. Accessed 11 April 2021

11. Essinger, J.: Jacquard's Web: How a Hand Loom Led to the Birth of the Information Age. Oxford University Press, Oxford (2007)

12. Liggett, S., Heald, K., Earnshaw, R.A., Thompson, E., Excell, P.S.: Collaborative research in art, design and new media-challenges and opportunities. In: Proceedings of Internet Technologies and Applications (2015). https://doi.org/10.1109/ITechA.2015.73174 56. Accessed 11 April 2021

13. Earnshaw, R.A., Liggett, S., Heald, K.: Interdisciplinary collaboration methodologies in art, design and media. In: Proceedings of International Conference on Internet Technologies and Applications, pp. 381-388. ISBN 978-0-946881-81-9, UK (2013). https://www. academia.edu/5779593/Interdisciplinary_Collaboration_Metho dologies_in_Art_Design_and_Media. Accessed 11 April 2021

14. Carroll, J.M. (ed.): Creativity and Rationale: Enhancing Human Experience by Design. Springer, London (2013)

15. Google acquires UK artificial intelligence start-up Deepmind, Guardian (2014). http://www.theguardian.com/technology/2014/ jan/27/google-acquires-uk-artificial-intelligence-startup-deepmind. Accessed 11 April 2021

16. Excell, P.S., Earnshaw, R.A.: The future of computing - the implications for society of technology forecasting and the kurzweil singularity. In: Proceedings of IEEE International Symposium on Technology and Society, Dublin, Ireland (2015). https://doi.org/10.1109/ ISTAS.2015.7439406. Accessed 11 April 2021

17. Dolinsky, M.: Facing experience: a painter's canvas in virtual reality. PhD thesis, Plymouth University (2014). http://pearl.plymouth. ac.uk/handle/10026.1/32042014dolinsky304581phdAnnotated.pdf. Accessed 11 April 2021

18. Mack, D., Ojalvo, H.E.: Real vs virtual: examining works of art online. New York Times, February 7 (2011). http://learning.blogs. nytimes.com/2011/02/07/real-vs-virtual-examining-works-of-artonline/? r=0. Accessed 11 April 2021

19. ITA15 Art Expo and Workshop (2015). https://sites.google.com/site/ ita15artexpoandworkshop/. Accessed 11 April 2021

20. ITA15 Carbon meets Silicon (2015). http://glyndwrpix.co.uk/carbo naugust2/. Accessed 11 April 2021

21. van Noorden, R.: Online collaboration: scientists and the social network. R. Nature. 512, 126-129 (2014). https://doi.org/10.1038/ $512126 \mathrm{a}$

22. Netscape Time: The Making of the Billion-dollar Start-up that Changed the World. J. Clark. St. Martin's Press, London (1999)
23. Deloitte Mobile Consumer Survey (2015). http://www2.deloitte. com/uk/en/pages/technology-media-and-telecommunications/artic les/mobile-consumer-survey.html. Accessed 11 April 2021

24. Ferrazzi, K.: How successful virtual teams collaborate. Harv. Bus. Rev. (2012). https://hbr.org/2012/10/how-to-collaborate-in-a-virtua. Accessed 11 April 2021

25. Curator, T., Anderson, C.: Crowd accelerated innovation. Wired (2010). http://www.wired.com/2010/12/ff_tedvideos/. Accessed 11 April 2021

26. Cunningham, S., Berry, D., Earnshaw, R.A., Excell, P.S., Thompson, E.: Multi-disciplinary creativity and collaboration: utilizing crowdaccelerated innovation and the internet. In: Proceedings of International Conference on Cyberworlds (2015). https://doi.org/10.1109/ CW.2015.29

27. KPMG: Accelerating Innovation: the Power of the Crowd. KPMG. https://www.kpmg.com/Ca/en/IssuesAndInsights/ArticlesPublica tions/Documents/Accelerating-Innovation-the-Power-of-the-Crowd. pdf. Accessed 11 April 2021

28. Brown, J.R., van Dam, A., Earnshaw, R.A., Encarnacao, J.L., Guedj, R.A., Preece, J., Shneiderman, B., Vince, J.: Human-centered computing, online communities and virtual environments. IEEE Comput. Graph. Appl. 19(6), 70-74 (1999). https://doi.org/10.1109/38. 799742

29. Brown, J.R., van Dam, A., Earnshaw, R.A., Encarnacao, J.L., Guedj, R.A.: Special report on human-centered computing, online communities and virtual environments. ACM SIGGRAPH Comput. Graph. 33(3), 42-62 (1999)

30. Earnshaw, R.A., Guedj, R.A., van Dam, A., Vince, J.A.: Frontiers of Human-Centered Computing, Online Communities and Virtual Environments. Springer, London (2001)

31. Asimov I.: Isaac Asimov asks how do people get new ideas? MIT Technol. Rev. 20 (2014). https://www.technologyreview.com/2014/ 10/20/169899/isaac-asimov-asks-how-do-people-get-new-ideas/

32. Guildford, J.P.: The Nature of Human Intelligence. McGraw-Hill, New York (1967)

33. Creativity. Wikipedia. https://en.wikipedia.org/wiki/Creativity\# Assessing_individual_creative_ability Accessed 11 April 2021

34. Creativity. Iowa State University. https://www.celt.iastate.edu/teach ing/teaching-format/14-creative-ways-to-engage-students/. Accessed 11 April 2021

35. Nowotny, H.: The Cunning of Uncertainty. Polity Press, Cambridge (2015)

36. Amabile T.: How to kill creativity. Harv. Bus. Rev. (1998). https:// hbr.org/1998/09/how-to-kill-creativity/ar/1 Accessed 11 April 2021

37. Jurgenson, N.: Digital dualism and the fallacy of web objectivity. Cyborgology (2011). https://thesocietypages.org/cyborgology/2011/ 09/13/digital-dualism-and-the-fallacy-of-web-objectivity/. Accessed 11 April 2021

38. Howard-Jones, P.: The Impact of Digital Technologies on Human Well Being. Nominet Trust (2011). https://static.lgfl.net/LgflNet/ downloads/online-safety/LGfL-OS-Research-Archive-2011-Nomin et-Impact-on-Wellbeing.pdf. Accessed 11 April 2021

39. Stahl, B.C., Timmermans, J., Flick, C.: Ethics of emerging information and communication technologies: on the implementation of responsible research and innovation. Sci. Public Policy 44(3), 369-381 (2017)

Publisher's Note Springer Nature remains neutral with regard to jurisdictional claims in published maps and institutional affiliations. 


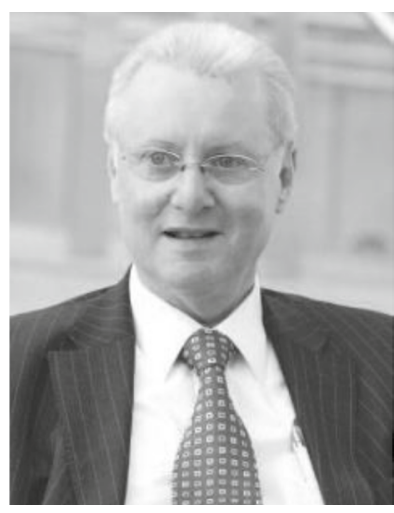

R. A. Earnshaw is full Professor of Electronic Imaging at the University of Bradford since 1995 (now Emeritus). He gained his $\mathrm{PhD}$ at the University of Leeds, $\mathrm{UK}$ and is a chartered engineer and chartered information technology professional. He was Dean of the School of Informatics (1999-2007) and Pro ViceChancellor (Strategic Systems Development) (2004-09). He has been a Visiting Professor at Illinois Institute of Technology,
George Washington University, USA, and Northwestern Polytechnical University, China. He has authored and edited 43 books on computer graphics, visualization, multimedia, design, and virtual reality, and published over 200 papers in these areas. https://www.bradford.ac.uk/ staff/raearnshaw. 